\title{
Multifocal VEP (mfVEP) reveals abnormal neuronal delays in diabetes
}

\author{
Brian E. Wolff • Marcus A. Bearse Jr. • \\ Marilyn E. Schneck • Shirin Barez • \\ Anthony J. Adams
}

Received: 1 April 2010/Accepted: 12 August 2010/Published online: 25 August 2010

(C) The Author(s) 2010. This article is published with open access at Springerlink.com

\begin{abstract}
This pilot study examined the diagnostic role of multifocal visually evoked potentials (mfVEP) in a small number of patients with diabetes. mfVEP, mfERG, and fundus photographs of both eyes of five patients with diabetes, three with nonproliferative diabetic retinopathy (NPDR) and two without NPDR were examined. Thirteen control subjects were also examined. Eighteen zones were constructed from the 60-element mfVEP stimulus array. mfVEP implicit time (IT) and amplitude (SNR) differences were tested between subject groups. We also examined whether there was a difference in function for patches with and without retinopathy in the NPDR group. Lastly, we compared mfVEP and mfERG results in the same patients. We found significant mfVEP IT differences between controls and all patients with diabetes, controls and diabetics without retinopathy, and between controls and diabetics with retinopathy. The subject groups did not differ significantly in terms of SNR. In the retinopathy group, ITs from zones with retinopathy were significantly longer than ITs from zones without retinopathy $(P=0.016)$. mfERG IT
\end{abstract}

Presentation at a conference: American Academy of Optometry, 2009, Orlando, Florida.

B. E. Wolff $(\square) \cdot$ M. A. Bearse Jr. ·

M. E. Schneck - S. Barez - A. J. Adams

Vision Science Program, School of Optometry, University

of California at Berkeley, 360 Minor Hall, Berkeley,

CA 94720-2020, USA

e-mail: Bewolff16@gmail.com was more frequently abnormal than mfVEP IT. In addition, mfERG hexagons were twice as likely to be abnormal if the corresponding mfVEP zone was abnormal $(P<0.05)$. mfVEP implicit times are significantly delayed in patients with diabetes even when there is no retinopathy. These cortical response results are similar, albeit considerably less abnormal, than those previously reported for retinal (mfERG) responses in patients with diabetes. A correlation exists between the location of abnormal mfERG hexagons and abnormal mfVEP zones.

Keywords Multifocal - Visually evoked potential . mfVEP $\cdot$ Diabetes - Diabetic retinopathy $\cdot$ mfERG

\section{Introduction}

Around the world, $6.6 \%$ percent of the adult population (285 million people) have diabetes, and an estimated 438 million people by the year 2030 will have diabetes [1]. In the United States, 8\% (23.6 million people) of the population have diabetes and another 57 million are prediabetic [2]. This has lead to 116 billion dollars of direct medical expenses in 2007 [2]. The cost for patients goes far beyond their pocket book. Diabetic retinopathy is the leading cause of blindness in the working age population (20-74 year olds), with up to 24,000 new cases of blindness each year in the United States [2]. 
Diabetic retinopathy is traditionally thought of as having a vascular etiology by way of increased blood glucose causing a breakdown in the blood retinal barrier. This leads to ischemia within the retina [2]. It is also known that diabetic retinopathy is caused by neurodegenerative abnormalities, perhaps independent of vascular abnormalities. These abnormalities are related to apoptosis, glial cell reactivity, microglial activation, and altered glutamate metabolism [3]. Our previous studies have shown that the multifocal electroretinogram (mfERG) is sensitive to these neurodegenerative or preclinical changes [4-7]. Patients with diabetes and no clinical signs of retinopathy can have significant mfERG P1 implicit time (IT) delays. Importantly, the IT delays are predictive of future nonproliferative diabetic retinopathy (NPDR) [4, 5, 7].

Given that the blood vessels and neural tissue throughout the body are affected by diabetes, it is reasonable to expect dysfunction to occur along the visual pathway upstream from the retina. MRI studies looking at patients with no history of stroke have shown $38 \%$ of patients with diabetes and $17 \%$ of nondiabetics to have ischemic lesions in the brain [8]. With this in mind, perhaps an even more sensitive measure of visual function in patients with diabetes could be performed with a measure of the visual pathway including V1 and possible extrastriate contributions. The visually evoked potential (VEP) provides such a measure. Previous studies with conventional full field and pattern reversal VEP have shown both amplitude and IT abnormalities in subjects with diabetes with and without retinopathy [9]. One previous study looked at mfVEP in patients with diabetes, focusing on relating mfVEP's to diabetic neuropathy [10]. Their study found that subjects with diabetes with neuropathy had greater mfVEP amplitude reduction than subjects without neuropathy. Bell et al. studied dichoptic multifocal pupillography in subjects with type 2 diabetes [11]. This novel technique of measuring a portion of the visual pathway found abnormalities when the duration of diabetes was beyond 10 years.

This pilot study gives a brief report on the diagnostic value of the mfVEP in five subjects with diabetes and 13 control subjects. The questions we sought to answer are (1) whether mfVEP IT and amplitude measures are abnormal in subjects with diabetes, (2) whether abnormalities occur without any retinopathy, (3) in what way do abnormalities compare to mfERG abnormalities, and (4) whether abnormalities are spatially associated with local NPDR.

\section{Methods}

\section{Subjects}

Five subjects with type 2 diabetes (three with mild to moderate NPDR in both eyes and two without NPDR in both eyes) and thirteen control subjects were examined. Subject groups were labeled in the following manner: the diabetes group as a whole as "DM", those with diabetic retinopathy as "NPDR", and those without diabetic retinopathy as "NoRet". Data collection consisted of measuring mfVEP on one visit, and taking fundus photographs and measuring the mfERG on a second visit within a one-month period. The mfERG was measured on all 5 diabetic subjects and on 10 of the 13 controls. Table 1 shows the demographic information. A retinal specialist graded the NPDR in the fundus photographs; this ophthalmologist was masked to the mfERG and mfVEP findings. All subjects were between the age of 25 and 65 with a mean age of $54.2 \pm 5.7$ and $43.6 \pm 12.1$ of
Table 1 Subject characteristics

\begin{tabular}{llllll}
\hline Subject & $\begin{array}{l}\text { Retinopathy } \\
(\mathrm{Y} / \mathrm{N})\end{array}$ & $\begin{array}{l}\text { Gender } \\
(\mathrm{M} / \mathrm{F})\end{array}$ & $\begin{array}{l}\text { Age } \\
\text { (years) }\end{array}$ & $\begin{array}{l}\text { DM type } \\
\text { (type) }\end{array}$ & $\begin{array}{l}\text { DM duration } \\
\text { (years) }\end{array}$ \\
\hline 1 & $\mathrm{Y}$ & $\mathrm{F}$ & 58 & 2 & 9 \\
2 & $\mathrm{Y}$ & $\mathrm{F}$ & 47 & 2 & 17 \\
3 & $\mathrm{Y}$ & $\mathrm{M}$ & 59 & 2 & 19 \\
4 & $\mathrm{~N}$ & $\mathrm{M}$ & 49 & 2 & 4 \\
5 & $\mathrm{~N}$ & $\mathrm{~F}$ & 58 & 2 & 4 \\
DM (mean $\pm \mathrm{SD})$ & $3 \mathrm{Y} / 2 \mathrm{~N}$ & $2 \mathrm{M} / 3 \mathrm{~F}$ & $54.2 \pm 5.7$ & 2 & $10.6 \pm 7.1$ \\
Control $($ mean $\pm \mathrm{SD})$ & $\mathrm{NA}$ & $5 \mathrm{M} / 8 \mathrm{~F}$ & $43.6 \pm 12.1$ & $\mathrm{NA}$ & $\mathrm{NA}$ \\
\hline
\end{tabular}


diabetics and controls, respectively. All had bestcorrected visual acuity of 20/25 or better and refractive error between \pm 6 diopters, were free of ocular disease (aside from NPDR), had no systemic diseases (aside from diabetes and hypertension) and no neurologic diseases or medications.

The nature of the study and any potential consequences of study participation were explained to the subjects, and written informed consent was obtained from all subjects before data collection. Procedures complied with the tenets of the Declaration of Helsinki and the University of California Committee for the Protection of Human Subjects approved the protocol.

\section{mfVEP Recording}

The scalp was cleaned with an alcohol swab followed by an abrasive paste. Once the scalp was cleaned, a gold cup electrode filled with conducting cream was placed onto the recording points. Electrode impedance was approximately $5 \mathrm{kOhm}$. We followed Hood et al's protocol of four electrodes on the back of the head and one on the forehead [12,13] (Fig. 1). An electrode on the forehead acted as a ground. On the back of the head, electrodes were placed as follows: one on the inion (which was the reference); another $4 \mathrm{~cm}$ above the inion; $4 \mathrm{~cm}$ to the left and $1 \mathrm{~cm}$ above the inion; and $4 \mathrm{~cm}$ to the right and $1 \mathrm{~cm}$ above the inion. An elastic wrap around the head kept the electrodes in place. Subjects were reclined

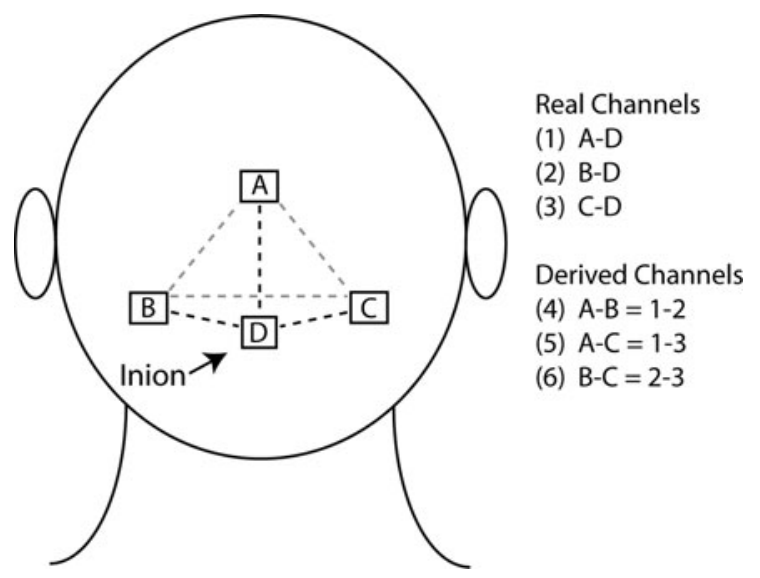

Fig. 1 Electrode placement schematic showing real mfVEP channels (1-3) and derived channels (4-6). Adapted from Hood et al. [12] slightly with an airline pillow behind the neck. Music was played to keep the subject alert.

The mfVEPs were recorded with a commercial system (VERIS 4.3; EDI Redwood City, CA). The signals were amplified 50,000 times. Low and high amplifier cut-offs were set to 3 and $100 \mathrm{~Hz}$, respectively. Frequencies $>35 \mathrm{~Hz}$ were digitally filtered out using Hood et al's Matlab program to improve signal to noise ratio (SNR) $[12,13]$. This was done only for the SNR analysis.

The stimulus consisted of a 60 -element checkerboard mfVEP array with a field diameter of $44.5 \mathrm{deg}$ displayed on a monochrome CRT (part of an eye camera/display/refractor unit) at a $75-\mathrm{Hz}$ frame rate. Each element contained eight black and eight white checks and was scaled for cortical magnification (Fig. 2a). The luminance values for the black and white checks were 2 and $200 \mathrm{~cd} / \mathrm{m}^{2}$, respectively. The background was $100 \mathrm{~cd} / \mathrm{m}^{2}$. Refractive error was corrected by focusing the stimulus eyepiece. Subjects fixated monocularly (the untested eye was patched) on a small cross in the center of the stimulus array. Fixation was constantly monitored with an infrared camera. Each element of the mfVEP array was independently modulated between checkerboard pattern reversals by a $2^{15}-1$ binary $m$-sequence to provide for 60 independent responses [12, 14-16]. Room lights were kept on throughout the testing period providing illumination approximately equal to the average luminance of the stimulus. Data were acquired in two 8-min recording sessions per eye. Each eight-min recording session consisted of 16 segments that were approximately $30 \mathrm{~s}$ in length. The order of recording was right eye, left eye, left eye, and right eye. Recording quality and fixation were monitored in real time. Segments with either large alpha-wave activity or loss of fixation were discarded and repeated.

\section{mfERG Recording}

mfERGs were recorded with the same commercial system as for the mfVEP. Subjects' pupils were dilated with $1.0 \%$ tropicamide and $2.5 \%$ phenylephrine, and $0.5 \%$ proparacaine was used to anesthetize the eye before placement of the Burian-Allen bipolar contact lens. A ground electrode was clipped to the right ear lobe, and the nontested eye was occluded. Both eyes of subjects with diabetes and left eyes of control subjects were tested. 

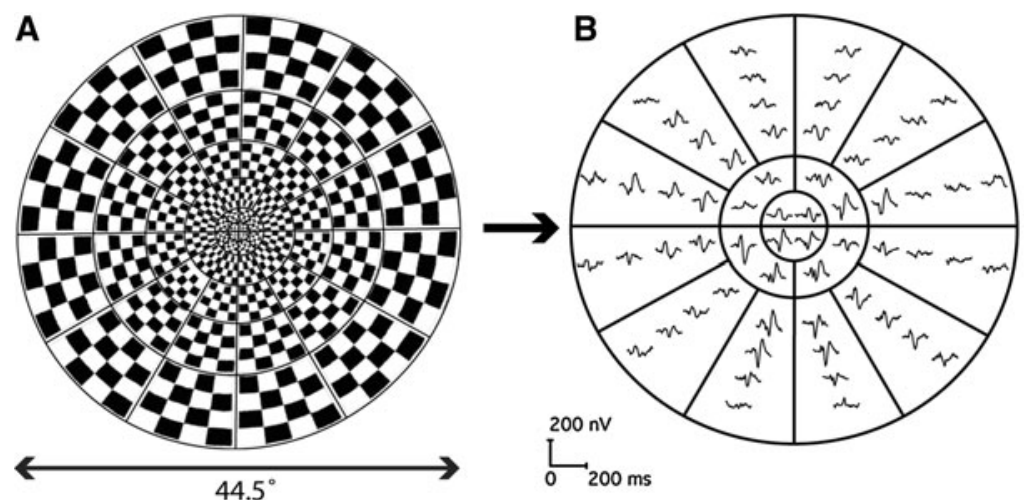

$\bigsqcup_{200 \mathrm{~ms}}$

Fig. 2 a The 60 element mfVEP array stimulus viewed by the patient. b The 60 response waveforms from a control subject. c The responses were then summed into 18 responses for better

The stimulus consisted of 103 scaled hexagonal elements consisting of a 45 deg field on the retina. Subjects fixated a small target in the center of the stimulus during the 8-min recording session. Each hexagon flickered between white and black throughout a $2^{15}-1$ binary $m$-sequence. Room lights were kept on during the testing session, providing an average luminance of the stimulus. Sixteen segments of approximately $30 \mathrm{~s}$ accounted for the 8-min total testing per eye. Fixation and quality of the recording were monitored in real time. Contaminated recordings were discarded and repeated. Band-pass filtering of $10-100 \mathrm{~Hz}$ with $100 \mathrm{~K}$ gain was used. See $\mathrm{Ng}$ et al. [17], 2008 for further details.

\section{Data Analysis}

The first slice of the second order mfVEP kernel was derived. This is the response that occurs when there is a pattern reversal in consecutive video frames $[12,15,16]$.

Eighteen retinal zones were constructed from the 60-element mfVEP array to allow for better SNR. The following process allowed us to retrieve the P-100 IT data. Signals obtained from both runs from channel one, which provided the highest SNR, were combined in VERIS. The 60 responses (Fig. 2b) were summed within 18 areas to increase SNR (Fig. 2c). P-100 ITs were manually and independently measured by three different observers within an 80-135 ms window following pattern reversal. The median of the three measurements was selected to establish P-100 IT. Finally, a Z-score was assigned signal to noise ratio in the array shown. The array with 18 summed response waveforms

for each raw IT of each zone based on control means and standard deviations. The SNR Z-scores were derived through Hood et al's mfVEP Matlab program [13]. This analysis provided $60 \mathrm{Z}$-scores based on the recording channel with the highest SNR. The first three channels were from the actual electrode placements. These 3 channels allowed for derivation of three additional channels. For example, channel 5 was derived by subtracting the recordings of channel 3 from those of channel 1, as shown in Fig. 1 [12, 13]. We then averaged the $Z$-scores within the 18 areas shown in Fig. 2c.

A superimposition process was used to determine whether there was a spatial association between the mfVEP array and the graded fundus photographs. First, the 18-zone mfVEP array was scaled to the fundus photograph grading sheet. The mfVEP data were then flipped along the horizontal axis to account for the difference between visual field and retinal orientation, and the locations of retinopathy lesions were noted. This was done independently for each eye.

The mfERGs were analyzed as reported in $\mathrm{Ng}$ et al. 2008 [17]. The local flash mfERG implicit times were measured by using a template scaling method [18]. Response templates were constructed from the mean local waveforms of 50 controls used in previous mfERG research [17]. Implicit times were derived as the time from focal flash onset to the first-positive peak (P1). IT Z-scores for diabetics and controls were derived from the mean and SD data from our 10 controls.

Spatial association of mfVEP IT with mfERG IT was also examined. The 103 hexagon mfERG array 
was scaled and superimposed onto the 18-zone mfVEP array. Some mfERG hexagons overlapped more than one mfVEP zone. These hexagons were split into the appropriate fractional unit to account for the overlapping. The percentage of abnormal mfERG IT $Z$-scores in each mfVEP zone were calculated for each diabetic and control subject.

\section{Statistical Analysis}

$Z$-scores $\geq 2$ (for IT) and $\leq-2$ (for $\mathrm{SNR}$ ) were considered abnormal (both $P<0.023$ ). The percentage of local abnormalities were calculated for mfVEP (IT and SNR) and mfERG (IT only).

Two-tailed Student $t$-tests were used to examine the differences between subject groups. A Bonferroni correction factor $(0.05 / 4)$ determined $P<0.0125$ as significant after multiple comparisons. Analysis was performed in two ways. First, for each subject group, every mfVEP response zone from all right and left eyes contributed to our analysis. This allowed for each individual to contribute 18 data points. Since our sample sizes between subject groups were unequal (i.e five subjects with diabetes versus 13 without), we also analyzed our data by creating an average right and left eye for each subject group. This was done by computing the average $Z$-score for each of the eighteen zones for each group.

Two-tailed Fisher Exact probability tests were used to investigate the association of mfVEP abnormalities with NPDR. Finally, differences between patches with and without retinopathy were investigated in eyes with NPDR using the two-tailed Student $t$-test.
A two-tailed Student $t$-test was also used to compare the percentage of abnormal mfERG area from abnormal mfVEP zones to the percentage of abnormal mfERG area from the nonabnormal mfVEP zones.

\section{Results}

Implicit Time

The percentage of locations with abnormal mfVEP P-100 IT were low and did not differ greatly among the subject groups. It was $6.7 \%$ OD and $5.6 \%$ OS in the DM group, 5.6\% OD and $2.8 \%$ OS in the NoRet group, $7.4 \%$ OD and $7.4 \%$ OS in the NPDR group, and $2.1 \%$ OD and $3.0 \%$ OS in controls (Fig. 3).

For mfERG P1 IT, there were many abnormalities in the diabetic groups. Furthermore, there were large and significant differences between diabetic groups and the nondiabetic control group. The percentage of locations with mfERG P1 IT abnormalities for all 103 retinal zones were $45 \%$ OD and $35 \%$ OS for the DM group, $28 \%$ OD and $3 \%$ OS for the NoRet diabetic group, $56 \%$ OD and $56 \%$ OS for the NPDR diabetic group, and $0.3 \%$ OS for the control group.

There were significant mfVEP IT differences between the following groups' averaged eyes: controls and DM $(P<0.0001 \mathrm{OD}$ and OS $)$; controls and NoRet $(P<0.02$ OD, $P<0.0005$ OS $)$; controls and NPDR $(P<0.0001$ OD and OS). The NoRet and NPDR groups did not differ significantly (Table 2 and Fig. 4). Results were similar when all
Fig. 3 Percentage of abnormalities is based on $Z$-score. IT abnormality is greater than or equal to a $Z$-score of +2 , and SNR (amplitude) abnormality is less than or equal to a $Z$-score of -2

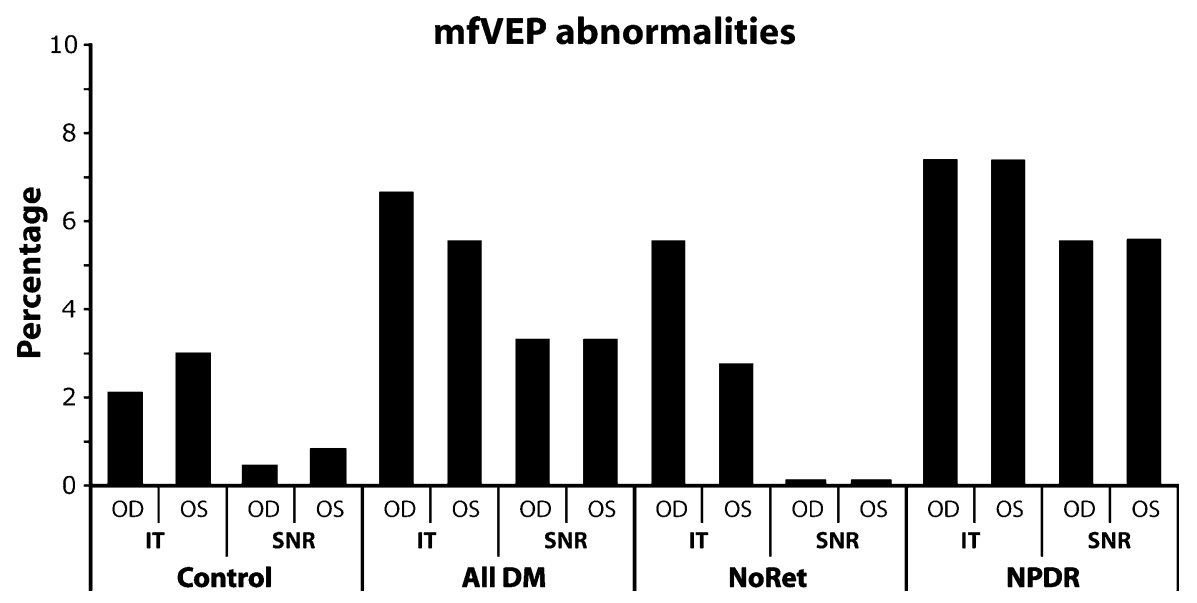


Table $2 P$-values of average eye group comparisons with two-tailed Student $t$-test

\begin{tabular}{|c|c|c|c|c|}
\hline & \multicolumn{2}{|l|}{ OD } & \multicolumn{2}{|l|}{ OS } \\
\hline & IT & SNR & IT & SNR \\
\hline Controls vs. all diabetics & $<0.001$ & 0.21 & $<0.001$ & 0.05 \\
\hline Controls vs. NoRet & 0.016 & 0.34 & $<0.001$ & 0.21 \\
\hline Controls vs. NPDR & $<0.001$ & 0.058 & $<0.001$ & 0.036 \\
\hline NoRet vs. NPDR & 0.20 & 0.034 & 0.50 & 0.42 \\
\hline
\end{tabular}

For mean $Z$-scores and standard errors, please see Fig. 4

Bold values indicate the significant $P$-values based on multiple comparisons

Fig. 4 a Mean IT Z-score value for each subject group. The mean and error bars are not seen for the controls because the values are very low. b Mean SNR $Z$-score value for each subject group. Average Z-scores for both plots are based off the average eye data
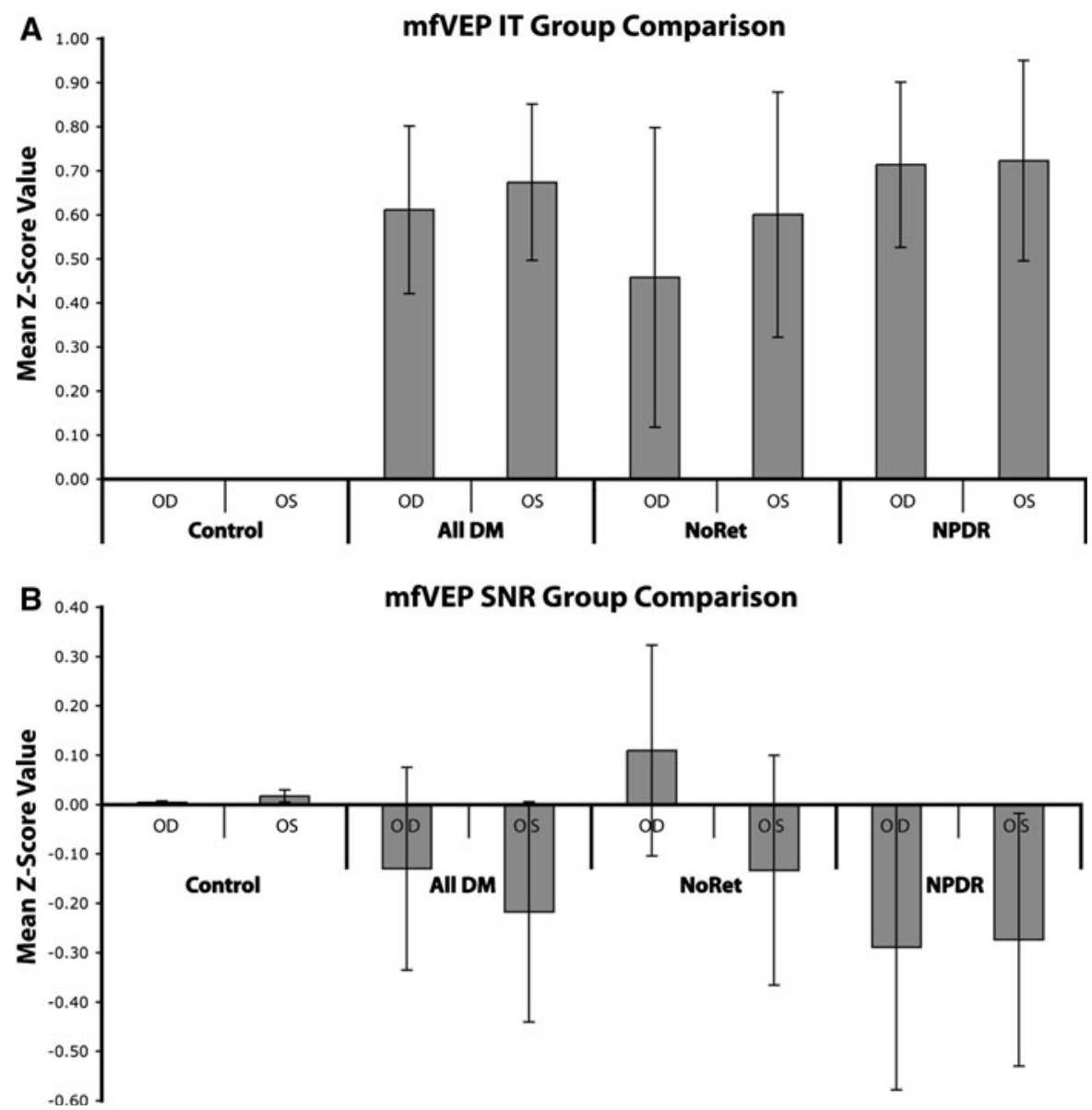

18 retinal zones for all eyes were examined, instead of averaging.

mfVEP IT abnormalities were not spatially associated with NPDR lesion sites $(P>0.10$ OD and OS). However, when patches with and without retinopathy in eyes with some NPDR were compared, there was a significant P100 IT difference with mean $Z$-scores of $0.93 \pm 0.83$ and $0.54 \pm 0.85$ in the patches with and without retinopathy, respectively $(P=0.016)$.

Analysis of spatial association between mfERG IT and mfVEP IT showed that the percentage of abnormal mfERG area from abnormal mfVEP zones to be nearly two times that found in normal mfVEP zones (67 and 34\%, respectively; $P<0.05$ ). Also, it was found that $27 \%$ of the abnormal mfVEP zones 
contained no mfERG abnormalities. Furthermore, $13 \%$ of the normal mfVEP zones contained $100 \%$ mfERG abnormalities.

\section{Amplitude}

The percentage of locations with abnormal SNR in the mfVEP were low and did not differ much among the subject groups. It was $3.3 \%$ OD and OS in the DM group, $0.0 \%$ OD and OS in the NoRet group, $5.6 \%$ OD and OS in the NPDR group, and 0.4\% OD and $0.9 \%$ OS in the controls (Fig. 3).

There were no significant differences between averaged eyes of the subject groups (Table 2 and Fig. 4). Results were similar when counting all 18 retinal zones for all eyes, instead of averaging.

mfVEP SNR abnormalities were not spatially associated with NPDR lesion sites $(P>0.50$ OD and OS). Also, there was no significant spatial association between mfVEP SNR abnormality and local NPDR within the NPDR eyes.

\section{Discussion}

The mfVEP ITs in subjects with diabetes are significantly different and delayed compared to controls. Diabetic subjects with and without NPDR show this delay. This suggests that retinopathy-free patients are showing early neurodegenerative changes occurring either at the level of the retina or upstream in the visual pathways that contribute to this cortical response.

The percentage of mfVEP P-100 IT abnormalities was up to $7.4 \%$ in the NPDR group and as low as $2.8 \%$ in the NoRet group (Fig. 3). The mfVEP amplitude measurement (SNR) appears to be less sensitive to the effects of diabetes than the IT measurement, which is similar to our retinal findings using the mfERG [7].

Our control and diabetes patient groups differed in age. However, although the patients with diabetes had an average age of $54.2 \pm 5.7$ years and the controls had an average age of $43.6 \pm 12.1$, it has been shown that age has minimal affect on mfVEP IT and amplitude [19, 20].

The IT of the mfERG is considerably more sensitive to the effects of diabetes than mfVEP IT, detecting up to 56 and $28 \%$ abnormalities in diabetics with and without NPDR, respectively. Given the limits of our small sample size, this is fairly consistent with our previously reported mfERG data, in which 49 and $20 \%$ abnormalities were detected in patients with and without NPDR, respectively [7].

Why are mfVEP responses less affected than mfERG responses in diabetes? First, the measurement of mfVEP signals is more complex. Even with proper conductance and adherence of the electrodes to the scalp, low SNR can be a limitation. Body fat can reduce the amplitude of the signal, muscle tension can create noise, and alpha waves elicited by sleep can create significant noise. Another factor contributing to the apparent lack of sensitivity may be our small sample size. But our data clearly show surprisingly large differences in the prevalence of abnormalities for the mfVEP and mfERG, not withstanding the small sample size.

We did not find a spatial association between NPDR and mfVEP abnormalities (either IT or SNR). A possible reason contributing to this was the mfVEP patch size relative to the NPDR lesion size. The lesions were much smaller than even our smallest mfVEP stimulus sectors. This issue would be difficult to address for any VEP recording from local retinal patches, especially in the periphery where large stimulus patches are required. Furthermore, averaging the 60 mfVEP sectors into 18 patches, which produced better SNR, further sacrificed spatial resolution. Small dot hemorrhages and even larger areas of edema or exudation appeared to have little local affect on the mfVEP waveforms. However, a separate analysis showed that zones with retinopathy were significantly delayed compared to zones without retinopathy in our patients with NPDR.

There was a higher percentage of abnormal mfERG hexagons in abnormal mfVEP zones compared to that found in normal mfVEP zones. This suggests that there is some propagation of retinal abnormalities into the cortex. It was also found that $27 \%$ of the abnormal mfVEP zones had $0 \%$ mfERG abnormalities. This could mean that the mfVEP is detecting pathology that is upstream from the retina. Finally, it was also found that $13 \%$ of normal mfVEP zones had $100 \%$ mfERG abnormalities. This is somewhat surprising and suggests that there is some compensation by the brain in these corresponding areas. One must, however, also consider that the mfVEP is less sensitive than the mfERG. These 
results are similar to the findings of Chen et al. 2006 [21] in other retinal diseases.

In conclusion, the mfVEP P100 implicit time in patients with diabetes, both with and without NPDR, is significantly delayed compared to nondiabetic controls. Importantly, even in patients without retinopathy, the mfVEP is detecting preclinical microvascular and/or neurodegenerative changes within or upstream from the retina. However, we found little or no spatial association of mfVEP abnormalities with NPDR, but significant difference between zones with and without retinopathy in our NPDR group. An important conclusion we make is that local mfVEP measure of delayed signals is less sensitive to the impact of diabetes than the mfERG. Further research with mfVEP measures, with larger study samples, is needed to more definitively explore any potential spatial association of the mfVEP abnormalities with local retinal lesions.

Acknowledgments AJA is supported by NIH EYRO102271. BEW is supported by T35 EY-07139-11, and California Chapter of the AAO Student Research Grant. Special thanks to Kevin Bronson-Castain, Ann Chang, Kavita Dhamdhere, Wendy Harrison, and Donna Lee.

Open Access This article is distributed under the terms of the Creative Commons Attribution Noncommercial License which permits any noncommercial use, distribution, and reproduction in any medium, provided the original author(s) and source are credited.

\section{References}

1. Silink M (2009) Diabetes atlas, 4th edn. International Diabetes Federation, USA

2. U.S. Department of Health, Human Services CfDCaP (2008) National diabetes fact sheet: general information and national estimates on diabetes in the United States, 2007. International Diabetes Federation, Atlanta, GA

3. Barber AJ (2003) A new view of diabetic retinopathy: a neurodegenerative disease of the eye. Prog Neuropsychopharmacol Biol Psychiatry 27:283-290

4. Han Y, Schneck ME, Bearse MA Jr, Barez S, Jacobsen CH, Jewell NP, Adams AJ (2004) Formulation and evaluation of a predictive model to identify the sites of future diabetic retinopathy. Invest Ophthalmol Vis Sci 45: 4106-4112

5. Han Y, Bearse MA Jr, Schneck ME, Barez S, Jacobsen $\mathrm{CH}$, Adams AJ (2004) Multifocal electroretinogram delays predict sites of subsequent diabetic retinopathy. Invest Ophthalmol Vis Sci 45:948-954
6. Fortune B, Schneck ME, Adams AJ (1999) Multifocal electroretinogram delays reveal local retinal dysfunction in early diabetic retinopathy. Invest Ophthalmol Vis Sci 40: 2638-2651

7. Bearse MA Jr, Adams AJ, Han Y, Schneck ME, Ng J, Bronson-Castain K, Barez S (2006) A multifocal electroretinogram model predicting the development of diabetic retinopathy. Prog Retin Eye Res 25:425-448

8. Biessels GJ, Koffeman A, Scheltens P (2006) Diabetes and cognitive impairment Clinical diagnosis and brain imaging in patients attending a memory clinic. J Neurol 253: 477-482

9. Ewing FM, Deary IJ, Strachan MW, Frier BM (1998) Seeing beyond retinopathy in diabetes: electrophysiological and psychophysical abnormalities and alterations in vision. Endocr Rev 19:462-476

10. Lovestam Adrian MKGL, Andersson G, Andreasson S (2007) Patients With Diabetic Retinopathy and Neuropathy Showed Lower Amplitudes on the Multifocal VEP Than Diabetic Patients With the Same Level of Retinopathy but No Neuropathy. Invest Ophthalmol Vis Sci 48:5010-5158

11. Bell A, James AC, Kolic M, Essex RW, Maddess T (2010) Dichoptic multifocal pupillography reveals afferent visual field defects in early type 2 diabetes. Invest Ophthalmol Vis Sci 51(1):602-608

12. Hood DC, Greenstein VC (2003) Multifocal VEP and ganglion cell damage: applications and limitations for the study of glaucoma. Prog Retin Eye Res 22:201-251

13. Hood DC, Zhang X, Hong JE, Chen CS (2002) Quantifying the benefits of additional channels of multifocal VEP recording. Doc Ophthalmol 104:303-320

14. Sutter EE (2001) Imaging visual function with the multifocal m-sequence technique. Vision Res 41:1241-1255

15. Hood DC, Zhang X, Greenstein VC, Kangovi S, Odel JG, Liebmann JM, Ritch R (2000) An interocular comparison of the multifocal VEP: a possible technique for detecting local damage to the optic nerve. Invest Ophthalmol Vis Sci 41:1580-1587

16. Hood DC, Odel JG, Winn BJ (2003) The multifocal visual evoked potential. J Neuroophthalmol 23:279-289

17. Ng JS, Bearse MA Jr, Schneck ME, Barez S, Adams AJ (2008) Local diabetic retinopathy prediction by multifocal ERG delays over 3 years. Invest Ophthalmol Vis Sci 49: 1622-1628

18. Hood DC, Li J (1997) A technique for measuring individual multifocal ERG records. In: Yager D (ed) Noninvasive Assessment of the Visual System, Trends in Optics and Photonics. Optical Society of America, Washington DC, pp 33-41

19. Li JJ, Xu L, Bai FG, Zhang RX, Wu XS, Sun XY (2005) Single channel multifocal VEP studies in normal Chinese. Zhonghua Yan Ke Za Zhi 41(4):355-363

20. Fortune B, Zhang X, Hood DC, Demirel S, Johnson CA (2004) Normative ranges and specificity of the multifocal VEP. Doc Ophthalmol 109(1):87-100

21. Chen JY, Hood DC, Odel JG, Behrens MM (2006) The effects of retinal abnormalities on the multifocal visual evoked potential. Invest Ophthalmol Vis Sci. 47(10): 4378-4385 